\title{
Sinonasal intestinal-type adenocarcinoma in the frontal sinus
}

\author{
Jaewoo Kim ${ }^{1}$, \\ Hak Chang ${ }^{1}$, \\ Euicheol C. Jeong ${ }^{2}$ \\ ${ }^{1}$ Department of Plastic and \\ Reconstructive Surgery, Seoul National \\ University College of Medicine, Seoul; \\ ${ }^{2}$ Department of Plastic Surgery, SMG- \\ SNU Boramae Medical Center, Seoul, \\ Korea
}

\section{INTRODUCTION}

Most adenocarcinomas that occur in the head and neck region originate from major or minor salivary glands. Sinonasal adenocarcinomas, however, are in the category of non-salivary tumors that originate from the surface epithelium of the nasal and paranasal sinuses. They are divided into intestinal- or nonintestinal-type, depending on the resemblance to gastrointestinal (GI) mucosa. The intestinal type is histologically composed of goblet, columnar, and mucus-producing cells which are usually indistinguishable from those of typical adenocarcinomas of the GI tract [1].

Sinonasal intestinal-type adenocarcinoma is a rare neoplasm. The overall incidence of intestinal-type adenocarcinoma is less than $1 \%$ of all neoplasms, and this tumor accounts for $1.4 \%$ of all tumors and less than $4 \%$ of malignancies in the sinonasal area $[2,3]$. Intestinal-type sinonasal adenocarcinomas occur primarily in the fifth to sixth decades of life, with a slight male

Correspondence: Euicheol C. Jeong

Department of Plastic Surgery, SMG-SNU Boramae Medical Center,

20 Boramae-ro 5-gil, Dongjak-gu, Seoul 07061, Korea

E-mail: ecjeong@snu.ac.kr

Received June 26, 2018 / Revised August 28, 2018 / Accepted August 31, 2018 preponderance and a strong association with occupational and environmental carcinogens such as wood and leather dust [4]. Exposure to wood dust has been reported to increase the risk of adenocarcinoma 900-fold [3].

Commonly presenting symptoms of sinonasal intestinal-type adenocarcinoma are nasal obstruction, epistaxis, and rhinorrhea. The tumor tends to be very locally aggressive but rarely metastasizes. Diagnosis with histological confirmation is important because it is frequently misdiagnosed as a salivary gland tumor. Additional immunohistochemical staining is helpful in classifying the grade and identifying the origin of the tumor. In addition, metastatic adenocarcinomas from the GI tract must be excluded by appropriate imaging or endoscopic studies [5]. Intestinal-type sinonasal adenocarcinomas are generally poorly differentiated, whereas nonintestinal tumors are histologically well differentiated. This morphological and immunohistochemical-based classification is valuable in predicting the prognosis of the disease and in determining the treatment plan [6].

The mainstay of the treatment strategy consists of aggressive surgical resection and radiation, or a combination of the two. Treatment failure and local recurrence are common; however, 
patients diagnosed with less extensive well differentiated tumors who are candidates for complete surgical resection reported improved outcomes [4,7]. We herein report a case of sinonasal adenocarcinoma of low grade intestinal-type to share our experience of dealing with this rare neoplasm with successful reconstruction.

\section{CASE REPORT}

A 63-year-old man reported a rapidly growing soft mass on his glabellar region for 4 months (Fig. 1). The painless soft mass was approximately $3 \times 2 \mathrm{~cm}$ with elevated skin. The patient was on medication for hypertension. On contrast computed tomography scan, we observed a $3.1 \times 2.6 \times 2.0 \mathrm{~cm}$ soft tissue mass at

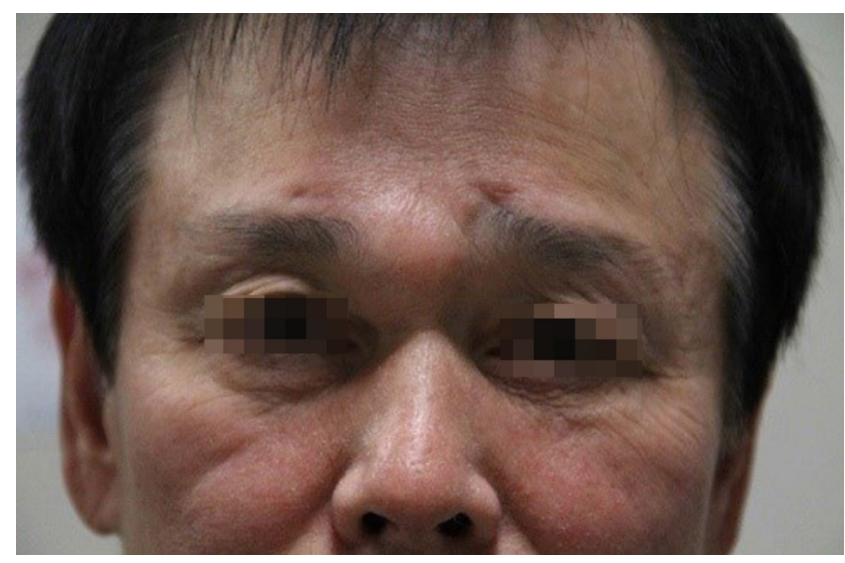

Fig. 1. A 63-year-old man with a painless soft mass on his glabellar region.

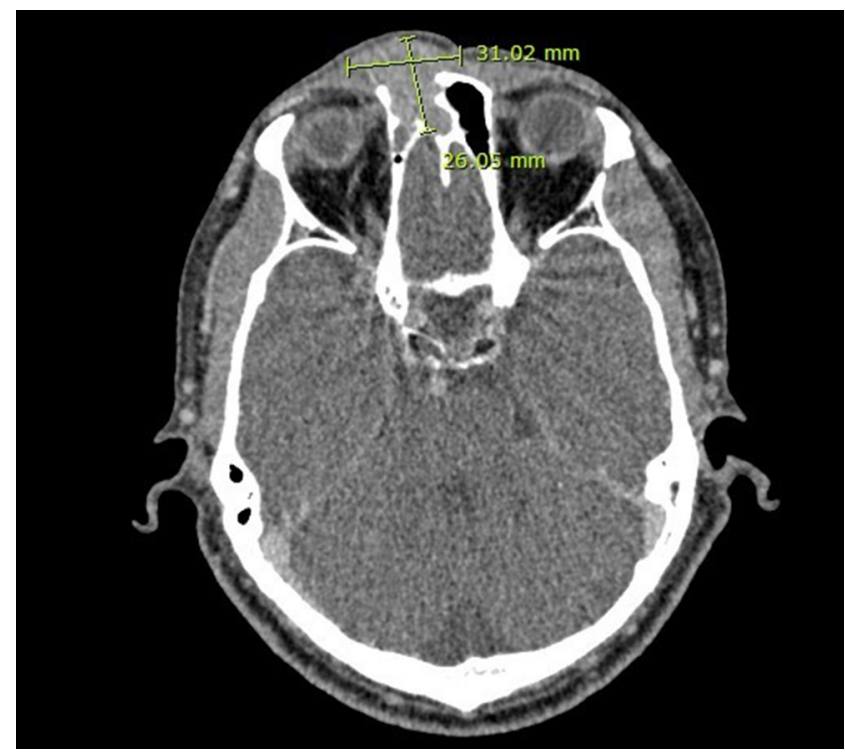

Fig. 2. Contrast computed tomography. A $3.1 \times 2.6 \times 2.0 \mathrm{~cm}$ soft tissue mass at the right fronto-ethmoidal sinus destructing the outer table of the frontal sinus wall. the right fronto-ethmoidal sinus destructing the outer table of the frontal sinus wall (Fig. 2). Our impression was a sinus-origin tumor. Thus, ultrasonography-guided fine needle aspiration was performed and a small, round cell malignancy was found on liquid-based cytology. For further evaluation, magnetic resonance imaging (MRI) (Fig. 3) and positron emission tomography scans were performed and no evidence of distant metastasis or metastatic regional lymph nodes were found. For histologic confirmation, we attempted a transcutaneous open biopsy through the elevated skin and the final pathology report was sinonasal intestinal-type adenocarcinoma (Figs. 4, 5). As this histologic finding could be related to a primary GI tract malignancy, we additionally screened for GI malignancies using endoscopy; no evidence of GI malignancy was found. Residual cancer

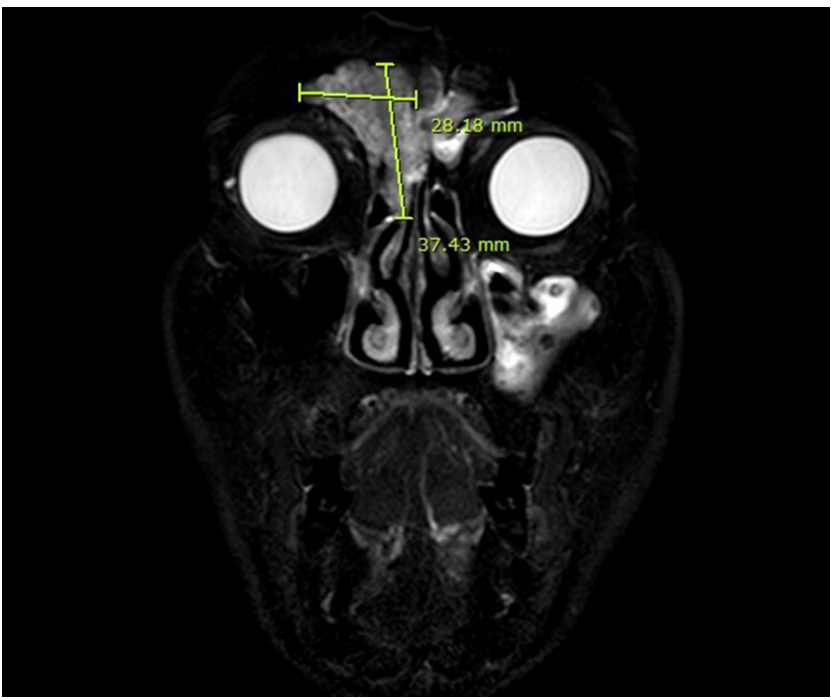

Fig. 3. Magnetic resonance imaging. Soft tissue enhancing mass at the right frontal sinus with extrusion through the right frontal sinus anterior wall. Suspicious extension to the left forehead and extension to the right ethmoid sinus and upper nasal soft tissue area was observed.

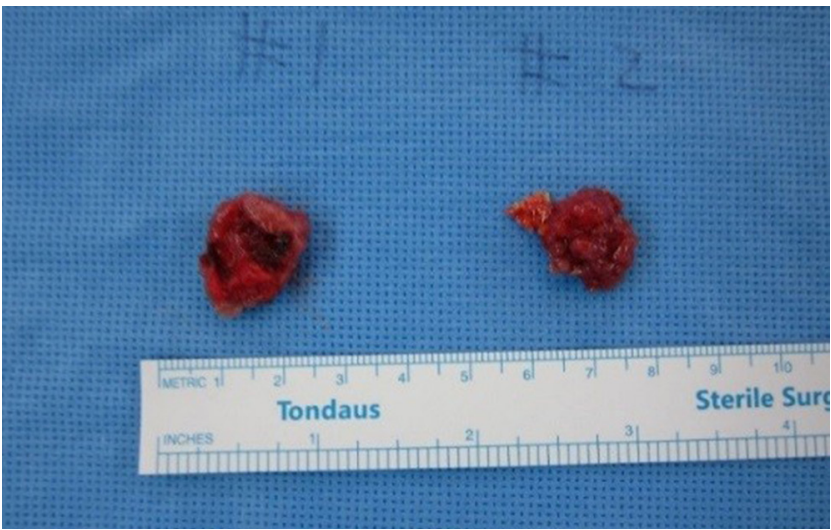

Fig. 4. Open biopsy gross photograph. Sinonasal intestinal-type adenocarcinoma (mass \#1 and \#2). 
and neck lymph node evaluation was verified by MRI, and there was also no evidence of neck lymph node metastasis. For surgical treatment, we widely excised the previous wound from the open biopsy and ablated the frontal sinus mucosa (Fig. 6). To cover the skin defect of the obliterated frontal sinus, immediate reconstruction with free anterolateral thigh flap was performed. The right superficial temporal artery and vein were used as the recipient vessels for microscopic anastomosis. There was no grossly residual tumor and the intraoperative surgical biopsy reported clear resection margins with no tumor involvement on the posterior mucosal wall of the frontal sinus. Immunohistochemical staining was performed to reveal sinonasal intestinal-type adenocarcinoma, favoring low-grade (Table 1 and Fig. 7). After consultation with radiation oncologists, we elected

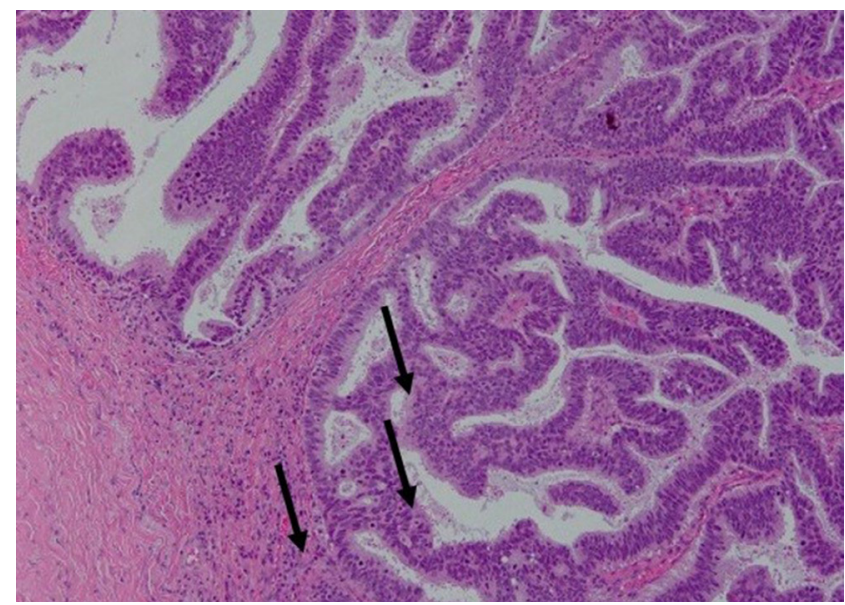

Fig. 5. Histopathologic finding. Sinonasal intestinal-type adenocarcinoma in the frontal sinus $(\mathrm{H} \& \mathrm{E}, \times 100)$. The arrows point some tumor cell invasion findings which are critical to diagnose malignancy.

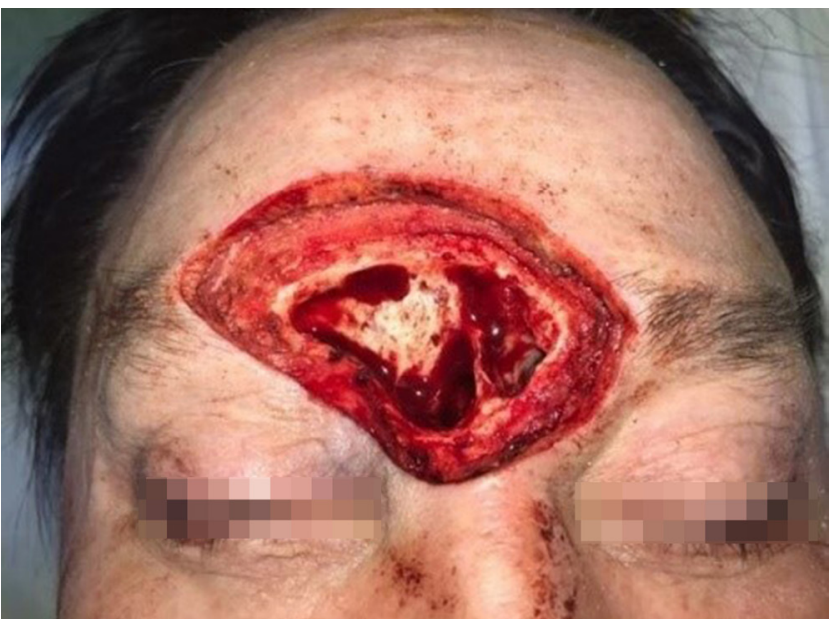

Fig. 6. Intraoperative photograph. Wide excision with removal of the frontal sinus posterior wall mucosa before immediate reconstruction. to skip postoperative adjuvant radiation therapy and instead closely observe the patient at the outpatient clinic. The wound healed completely (Fig. 8). The patient is currently at 6 months after surgery without signs of recurrence at the radiologic follow-up.

Table 1. Immunohistochemical staining profiles of pathologic report

\begin{tabular}{ll}
\hline Marker & \multicolumn{1}{c}{ Reactivity } \\
\hline CDX2 & Negative \\
p53 & Negative \\
Ki-67 & Positive, nearly 90\% \\
Cytokeratin 7 & Positive \\
Cytokeratin 20 & Negative \\
\hline
\end{tabular}

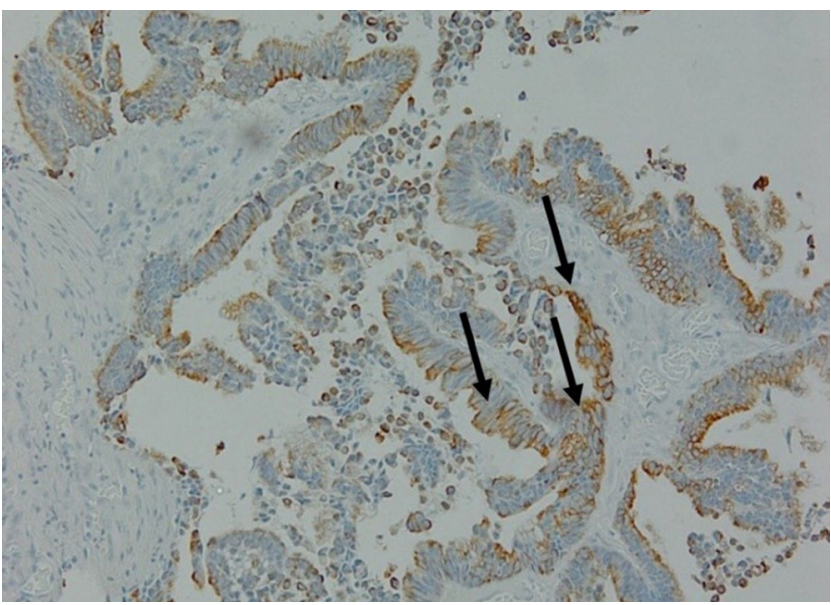

Fig. 7. Immunohistochemical staining. Sinonasal adenocarcinoma, favoring low-grade. Ki-67 positive, nearly $90 \%(\times 200)$. The arrows point some stained cells which are $\mathrm{Ki}-67$ positive.

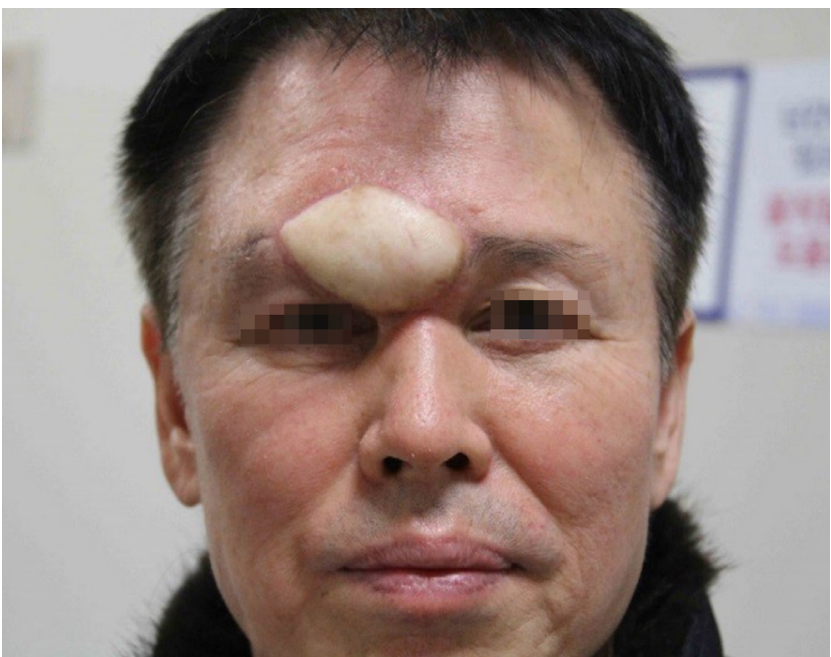

Fig. 8. Postoperative follow-up photograph at the outpatient clinic after 76 days. 


\section{DISCUSSION}

Sinonasal intestinal-type adenocarcinoma is a rare neoplasm. It generally appears to be a poorly differentiated tumor, with aggressive behavior and high local recurrence rates [3]. Franchi et al. [4] reviewed the outcomes of 41 patients with intestinal-type adenocarcinoma followed for more than 108 months; approximately $46 \%$ of patients had local recurrence of the tumor after initial treatment, and 56\% ultimately died from the disease. In our case, however, the patient was diagnosed with sinonasal intestinal-type adenocarcinoma, favoring low-grade. This is a much rarer situation [6] in which a relatively better prognosis can be expected.

The patient primarily visited the department of plastic surgery with a painless glabellar mass being his chief complaint. This is somewhat different from other sinonasal intestinal-type adenocarcinoma reports. Typically, the chief complaint is nasal obstruction and the patient primarily visits the department of otorhinolaryngology. This unusual presentation is meaningful, as it implies that plastic surgeons may need to consider sinonasal intestinal-type adenocarcinoma as a differential diagnosis when a patient presents with a painless paranasal mass.

The patient was a 63-year-old man who had age and sex risks for sinonasal neoplasms, but no history of exposure to environmental carcinogens, such as leather or wood dust. After a series of preoperative evaluations, we postulated that complete surgical resection was possible, and postoperative adjuvant radiation therapy was not planned considering the risks and benefits. As the targeted region of irradiation was the periorbital area, the possibility of cataracts on both eyes was essentially $100 \%$; additionally, several side effects of radiation toxicity, including keratoconjunctivitis sicca, radiation retinopathy, and radiation optic neuropathy, were expected. Since the tumor was well differentiated with a relatively indolent character and we had managed to achieve total resection with clear surgical margins, we decided to closely follow up the patient instead of taking the potential risks associated with radiation.

For reconstruction, we used the free anterolateral flap from the thigh. It is a less common choice than the radial forearm flap in forehead reconstruction. We chose the thigh as a donor because the patient did not want any scars or discomfort in his upper extremities. Tissue expansion and local flap for an improved cosmetic result were not considered because of the local recurrence risk. Nevertheless, had the patient desired a better cosmetic result, we may have considered delaying reconstruc- tion after the immediate postoperative period requiring close follow-up. The skin paddle of the anterolateral thigh flap was partially de-epithelialized to match the size of the skin defect. The soft tissue beneath filled up the defect made after obliteration of the denuded sinus. By using this means, while minimizing cosmetic deterioration during the close follow-up period, we could also leave open the possibility for additional procedures to improve cosmesis such as staged excision or tissue expansion of the substituted skin portion.

\section{CONFLICT OF INTEREST}

No potential conflict of interest relevant to this article was reported.

\section{PATIENT CONSENT}

The patients provided written informed consent for the publication and the use of their images.

\section{REFERENCES}

1. Franquemont DW, Fechner RE, Mills SE. Histologic classification of sinonasal intestinal-type adenocarcinoma. Am J Surg Pathol 1991;15:368-75.

2. Boor A, Jurkovic I, Dudrikova K, Kavecansky V, Friedmann I. Intestinal-type sinonasal adenocarcinoma: a sporadic case. J Laryngol Otol 1996;110:805-10.

3. Sklar EM, Pizarro JA. Sinonasal intestinal-type adenocarcinoma involvement of the paranasal sinuses. AJNR Am J Neuroradiol 2003;24:1152-5.

4. Franchi A, Gallo O, Santucci M. Clinical relevance of the histological classification of sinonasal intestinal-type adenocarcinomas. Hum Pathol 1999;30:1140-5.

5. McKinney CD, Mills SE, Franquemont DW. Sinonasal intestinal-type adenocarcinoma: immunohistochemical profile and comparison with colonic adenocarcinoma. Mod Pathol 1995;8: 421-6.

6. Sayilgan AT, Kamali G, Ozcan D, Emre F, Hatipoglu A. Low grade sinonasal adenocarcinoma. Turk Patoloji Derg 2012;28: 67-71.

7. Alessi DM, Trapp TK, Fu YS, Calcaterra TC. Nonsalivary sinonasal adenocarcinoma. Arch Otolaryngol Head Neck Surg 1988;114:996-9. 